\title{
The investigation of multi-walled carbon nanotubes dispersion and its influence on rheological properties of cement systems
}

\author{
Gintautas Skripkiunas $^{1}$, Grigory Yakovlev $^{2}$, and Ekaterina Karpova ${ }^{1, *}$ \\ ${ }^{1}$ Vilnius Gediminas Technical University, Department of Building Materials and Fire Safety, LT- \\ 10223 Vilnius, Lithuania \\ ${ }^{2}$ Kalashnikov Izhevsk State Technical University, Department of Geo-Engineering and Construction \\ Materials, 426069 Izhevsk, Russia
}

\begin{abstract}
The current research is devoted to the studying of multi-walled carbon nanotubes (MWCNT) dispersion and its influence on the rheological properties of cement pastes. The $\mathrm{pH}$ and electrical conductivity test, zeta potential measurements, particle size analysis were performed for characterization of MWCNT dispersion. The MWCNT dispersion were tested during 1 month and presented the decrease of $\mathrm{pH}$ level from 8.89 to 8.02 , increase of electrical conductivity from 2.00 to $2.75 \mathrm{mS} / \mathrm{cm}$. The particle size analysis did not reveal the significant changes during 1 month of storage. The rheological test of nanomodified cement pastes were performed for concentration of MWCNT dispersion in the range from 0 to $0.5 \%$ by weight of cement. The decrease of yield stress by $30.7 \%$ and increase of plastic viscosity by $29.6 \%$ were obtained for cement paste modified by MWCNT dispersion in dosage of $0.25 \%$ by weight of cement. The changes in flow behaviour were established for cement pastes tested in 5 min after mixing and were not observed for cement pastes tested after 120 min of mixing.
\end{abstract}

\section{Introduction}

The investigation of rheological properties of cement systems enables to predict and manage by the properties of concrete mixture and cement mortar. This field of research is becoming more relevant with the development of new generation concrete such as selfconsolidating (SCC), high-performance (HPC) and ultra-high performance concrete (UHPC). The development of new concrete technologies and fields of concrete application are required the understanding of rheological behavior of cement systems as well [1-5].

The composition of modern concrete mixtures consists of different types mineral and chemical admixtures. The changes in rheology of cement pastes with admixtures get the ability to clarify the mechanisms of their actions which determine their effectiveness in cement systems.

\footnotetext{
* Corresponding author: ekaterina.karpova@vgtu.lt
} 
For example, in the research [6] authors investigated the dilatancy of cement paste with replacement of cement mass by micro silica. The replacement by $9 \%$ of micro silica leads to volume change of cement paste from $1.22 \%$ to $0 \%$ and the reduce of dilatancy.

The plasticizing admixtures, as a type of chemical admixtures, are used to disperse the cement particles in volume of concrete mixture and improve the workability of concrete. The investigation of authors [7] revealed that the decrease of yield stress in cement systems for polycarboxylate plasticizer caused by steric effect of action mechanism. At the same time, the cluster formation of polycarboxylate plasticizer, caused by polymer chains, increase the viscosity of cement systems. The better plasticizing effect is achieved by increase of zeta potential, decrease of surface tension and steric effect determined by molecular parameters of monomers [8].

Along with mineral and chemical additives for concrete, the nanomodification of cement systems is a perspective tool to manage by the properties of cement systems in fresh and hardened state.

Nanomodification of cement systems by carbon nanotubes (CNT) demonstrates a positive effect on a number properties of cement composites [9-12]. However, the tendency of nanostructures to agglomeration limits their application in construction practise [13], [14]. Mainly, the properties of cement composites with nanostructures are depended on the quality of CNT dispersion [15-17]. Despite the significant number of publications devoted to nanomodification of cement systems, this topic remains relevant due to unclear mechanisms of interaction between CNT and cement system. The comprehensive approach is required to study the cement systems modified by CNT.

This paper focuses on the investigation of MWCNT dispersion properties during storage and its influence on rheological properties of cement pastes.

\section{Materials and methods}

\subsection{Materials}

The cement CEM I 42.5 R (supplied by "Akmenes Cementas", Lithuania) conforming to EN 197-1 was used as a binder. The mineral composition of the cement is presented in Table 1. The physical and mechanical properties of the cement are listed in Table 2.

Table 1. Mineral composition of cement.

\begin{tabular}{|c|c|}
\hline Component & Amount, $\%$ \\
\hline Tricalcium silicate $\left(\mathrm{C}_{3} \mathrm{~S}\right)$ & 57.9 \\
\hline Dicalcium silicate $\left(\mathrm{C}_{2} \mathrm{~S}\right)$ & 15.6 \\
\hline Tricalcium aluminate $\left(\mathrm{C}_{3} \mathrm{~A}\right)$ & 7.5 \\
\hline Tetracalcium aluminoferrite $\left(\mathrm{C}_{4} \mathrm{AF}\right)$ & 11.9 \\
\hline
\end{tabular}

Table 2. Physical and mechanical properties of cement

\begin{tabular}{|c|c|}
\hline Property & Value \\
\hline 2-day compressive strength, $\mathrm{MPa}$ & $28 \pm 2$ \\
\hline 28-day compressive strength, $\mathrm{MPa}$ & $55 \pm 3$ \\
\hline Initial setting time, min & 180 \\
\hline Final setting time, $\mathrm{min}$ & 225 \\
\hline Volume stability, $\mathrm{mm}$ & 1.0 \\
\hline Water consumption, $\%$ & 26.5 \\
\hline Residue on the $90 \mu \mathrm{m}$ sieve, $\%$ & 1.5 \\
\hline Specific surface, $\mathrm{cm}^{2} / \mathrm{g}$ & 4000 \\
\hline
\end{tabular}


Potable water according to EN 1008 was used for preparation of cement pastes.

The MWCNT dispersion was prepared from the masterbatch pellets "Graphistrength CW 2-45" produced by the company "Arkema" (France). The masterbatch contains $45 \mathrm{wt}$. $\%$ of MWCNT and $55 \mathrm{wt}$ \% of carboxymethylcellulose (CMC). The MWCNT length was equal to $0.1-10 \mu \mathrm{m}$ and diameter equal to $15-20 \mathrm{~nm}$. The concentration of "Graphistrength CW 2-45" in the dispersion was taken equal to $1.0 \%$.

\subsection{Methods}

The MWCNT dispersion was prepared according to the following procedure. The masterbatch "Graphistrength CW 2-45" were immersed in hot water with temperature about $75^{\circ} \mathrm{C}$ and stirred by standard mixer agitation about $1000 \mathrm{rpm}$ during $15 \mathrm{~min}$. After that, the dispersion was subjected to ultrasonication for a duration of $6 \mathrm{~min}$. The ultrasonication was performed by Bandelin Sonopuls HD 3400 ultrasonic homogenizer $(400 \mathrm{~W}, 20 \mathrm{kHz})$ with probe VS 200 T (Ø 25mm, amplitude - $82 \mu \mathrm{m})$.

The dispersion was evaluated by $\mathrm{pH}$-meter Mettler-Toledo EL20 with limits of error \pm $0.01 \mathrm{pH}$; electrical conductivity meter Mettler-Toledo EL30 tests with limits of error \pm 0.5 $\%$. Particle size analysis of the dispersion was performed by particle size and zeta potential analyser DelsaNano C, Beckman Coulter with resolution of measurements placed from 0.6 $\mathrm{nm}$ to $7 \mu \mathrm{m}$.

The cement pastes with composition presented in Table 3 were prepared for rheological test.

Table 3. Content of materials for $1 \mathrm{~m}^{3}$ in tested cement pastes.

\begin{tabular}{|c|c|c|c|c|c|c|}
\hline Specimen & $\begin{array}{c}\text { Cement, } \\
\mathrm{kg}\end{array}$ & $\begin{array}{c}\text { Water, } \\
\mathrm{kg}\end{array}$ & $\begin{array}{c}\text { W/C } \\
\text { ratio }\end{array}$ & $\begin{array}{c}\text { MWCNT, } \\
\mathrm{kg}\end{array}$ & $\begin{array}{c}\text { MWCNT } \\
\text { dispersion, } \\
\mathrm{kg}\end{array}$ & $\begin{array}{c}\text { MWCNT } \\
\text { dispersion, } \\
\text { \% by weigh of } \\
\text { cement }\end{array}$ \\
\hline D0 & 935 & 281 & 0.30 & 0 & 0 & 0 \\
\hline D1 & 935 & 279 & 0.30 & 0.01 & 1.17 & 0.125 \\
\hline D2 & 935 & 278 & 0.30 & 0.02 & 2.34 & 0.25 \\
\hline D3 & 935 & 276 & 0.30 & 0.05 & 4.68 & 0.50 \\
\hline
\end{tabular}

The MWCNT dispersion was dissolved in potable water. Water together with MWCNT dispersion was added to the cement and cement paste was mixed for $180 \mathrm{~s}$ at high speed by mixer.

Rheological properties of cement pastes were tested in different time after pastes mixing: 5, 30, 60 and $120 \mathrm{~min}$. The rheology tests were carried out at temperature $20 \pm 2{ }^{\circ} \mathrm{C}$. The rotational rheometer Rheotest RN 4.1 with coaxial cylinders was used for rheological tests. The scheme of cylinder measuring system of rheometer is presented in Figure 1.

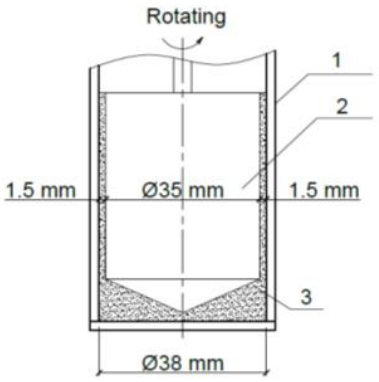

Fig. 1. Scheme of cylinder measuring system Rheotest RN 4.1. 
The assembled measuring cup (1) with coupling is poured by cement paste (3), and fixed in the equipment stand. The rotating cylinder rotor (2) is placed inside the measuring cup (1) with gap between them about $1.5 \mathrm{~mm}$ and rotates during the test. Shear rate ranged from $100.0 \mathrm{~s}^{-1}$ to $0.1 \mathrm{~s}^{-1}$ during testing time. The shear rate mode presented in Figure 2 was used in the current experiments.

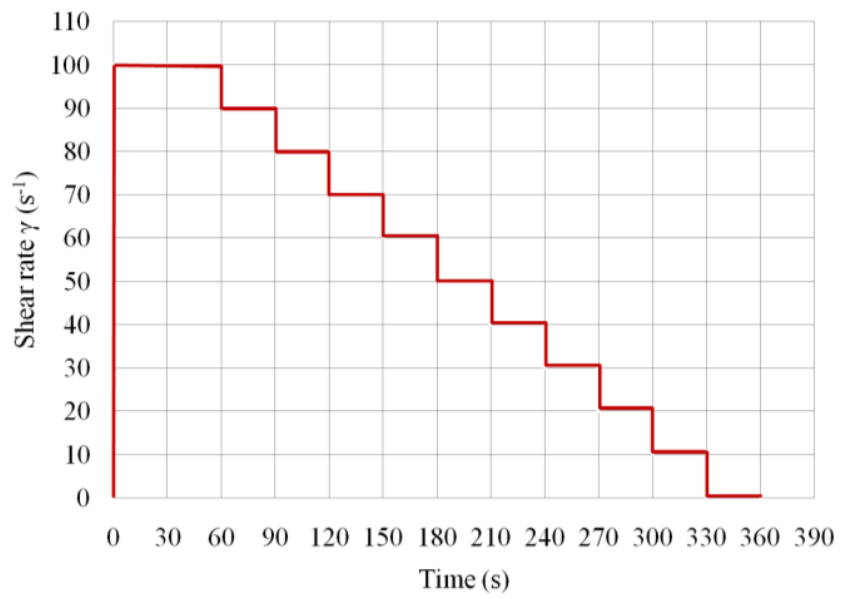

Fig. 2. Shear rate mode of rheological test.

Cement pastes after mixing between tests were placed in the plastic containers and carried in the laboratory conditions with temperature $20 \pm 2{ }^{\circ} \mathrm{C}$ and relative humidity not less than $65 \%$. The cement pastes were premixed before rheological test by hands using metallic scoop for $1 \mathrm{~min}$.

\section{Results and discussion}

Figure 3a shows the changes in $\mathrm{pH}$ of MWCNT dispersion during the storage, $\mathrm{pH}$ level was decreased from 8.89 to 8.02 . In other words, the dispersion medium becomes more acidic during the storage.

Figure $3 \mathrm{~b}$ demonstrates the changes in electrical conductivity of MWCNT dispersion during the storage. Electrical conductivity was increased from 2.00 to $2.75 \mathrm{mS} / \mathrm{cm}$.

The results presented in Figure $3 a, b$ can be explained by the influence of MWCNT on the physic-chemical processes which take place in water medium of dispersion.

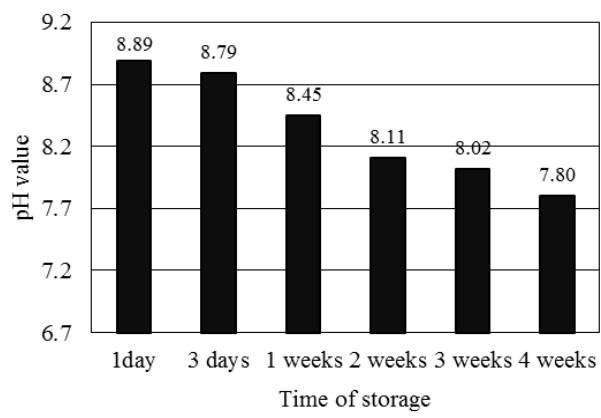

a)

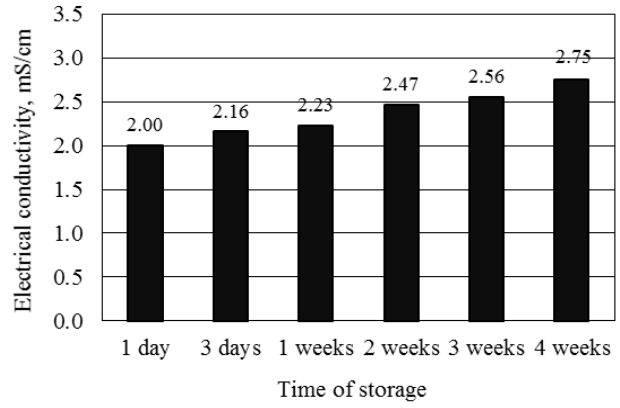

b)

Fig. 3. $\mathrm{pH}$ level (a) and electrical conductivity (b) of MWCNT dispersion during the storage. 
The particle size analysis of MWCNT dispersion after the homogenization revealed that the diameter of MWCNT dispersion particles was varied from 119 to $1070 \mathrm{~nm}$. The average diameter of particles was established equal to $294 \mathrm{~nm}$. The results of particle size analysis are presented in Fig.4.

The test of MWCNT dispersion after 1 month of storage presents variation of particle sizes in the range from 82 to $677 \mathrm{~nm}$ and average diameter about $270.3 \mathrm{~nm}$. It means that obtained MWCNT dispersion keeps the stability during 1 moth of storage, however the changes in $\mathrm{pH}$ and electrical conductivity demonstrate the significant changes during the storage which can be linked with possible coagulation processes in the volume of the MWCNT dispersion.

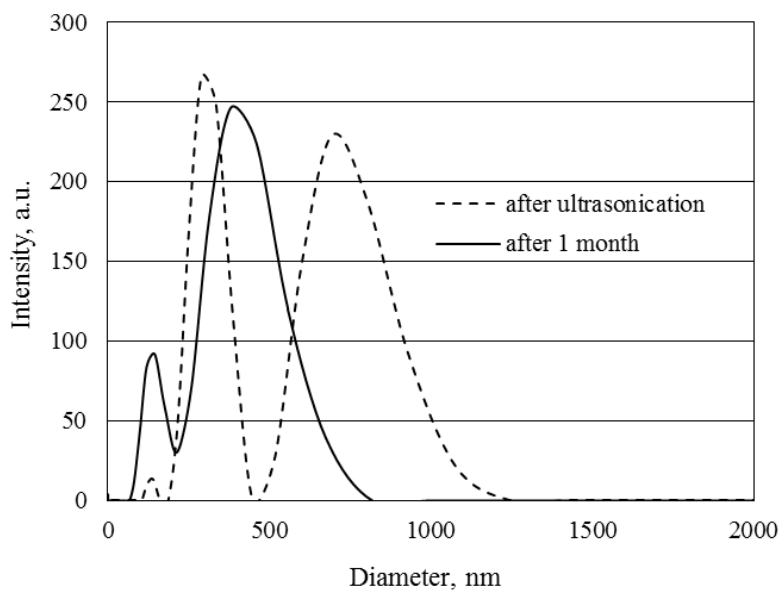

Fig. 4. The particle size distribution by intensity of MWCNT dispersion after ultrasonication and after 1 month of storage.

The experimental rheological flow curves are presented in Fig. 5 and Fig. 6 after 5 and $120 \mathrm{~min}$ of testing, respectively.

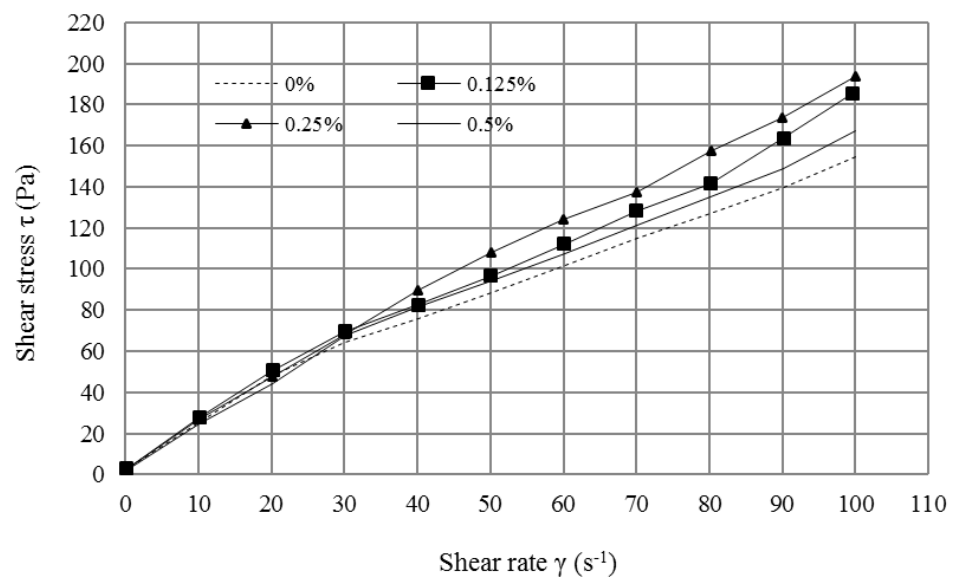

Fig.5. Flow curves of nanomodified cement pastes after 5 min of mixing. 


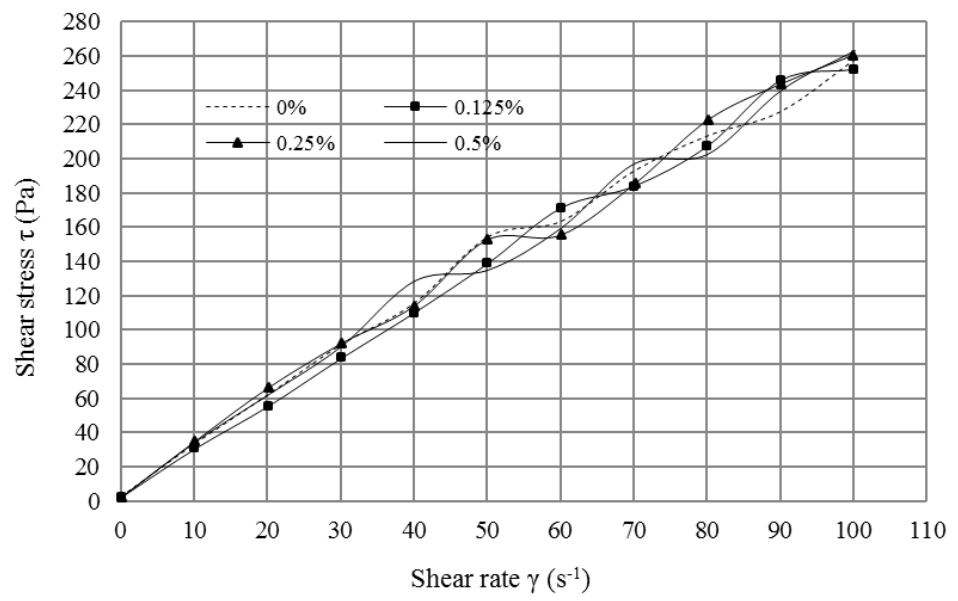

Fig.6. Flow curves of nanomodified cement pastes after 120 min of mixing.

The yield stress $\left(\tau_{0}\right)$ and plastic viscosity $\left(\mu_{\mathrm{pl}}\right.$.) of cement pastes were determined in the course of linear approximation of flow curve (dependence between shear stress $(\tau)$ and shear rate $(\gamma)$ in the range from 0.1 to $100 \mathrm{~s}^{-1}$ based on Bingham model, which is expressed by Equation 1:

$$
\tau=\tau_{0}+\mu_{p l} \cdot \gamma
$$

where: $\tau$ - shear stress, $\mathrm{Pa}$;

$\tau_{0}$ - yield stress of the cement paste, $\mathrm{Pa}$;

$\mu_{\text {pl. }}$ - plastic viscosity of the cement paste, Pa.s;

$\gamma$ - shear rate, $\mathrm{s}^{-1}$.

The equations obtained during the approximation of experimental curves according to Bingham model are presented in Table 4.

Table 4. Flow curves equations based on Bingham model.

\begin{tabular}{|c|c|c|}
\hline Specimen & After 5 min & After 120 min \\
\hline D0 $(0 \%)$ & $\tau=13.90+1.433 \gamma$ & $\tau=12.22+2.507 \gamma$ \\
\hline D1 $(0.125 \%)$ & $\tau=11.71+1.569 \gamma$ & $\tau=5.934+2.568 \gamma$ \\
\hline D2 $(0.25 \%)$ & $\tau=11.05+1.707 \gamma$ & $\tau=10.76+2.561 \gamma$ \\
\hline D3 $(0.5 \%)$ & $\tau=9.636+1.857 \gamma$ & $\tau=10.95+2.536 \gamma$ \\
\hline
\end{tabular}

The flow behaviour of cement pastes modified by MWCNT dispersion was changed in comparison with reference cement paste. The increase of shear stress and plastic viscosity of nanomodified cement pastes is observed in Fig. 5 in the range of shear rate from 0.1 to $100 \mathrm{~s}^{-1}$ for $5 \mathrm{~min}$ after mixing. The maximum increase of shear stress was identified for cement paste modified by MWCNT dispersion in dosage $0.25 \%$ by weight of cement.

However, as it seen in Fig. 6, shear stress and plastic viscosity are not changed significantly for nanomodified cement pastes in the range of shear rate from 0.1 to $100 \mathrm{~s}^{-1}$ for 120 min after mixing.

The changes in yield stress and plastic viscosity values for cement pastes with different dosage of MWCNT for 5 and 120 min after mixing with respect to the reference sample are presented in Figure 7, 8. The values of yield stress and plastic viscosity were obtained based on approximation of experimental curves according to the Bingham model. 


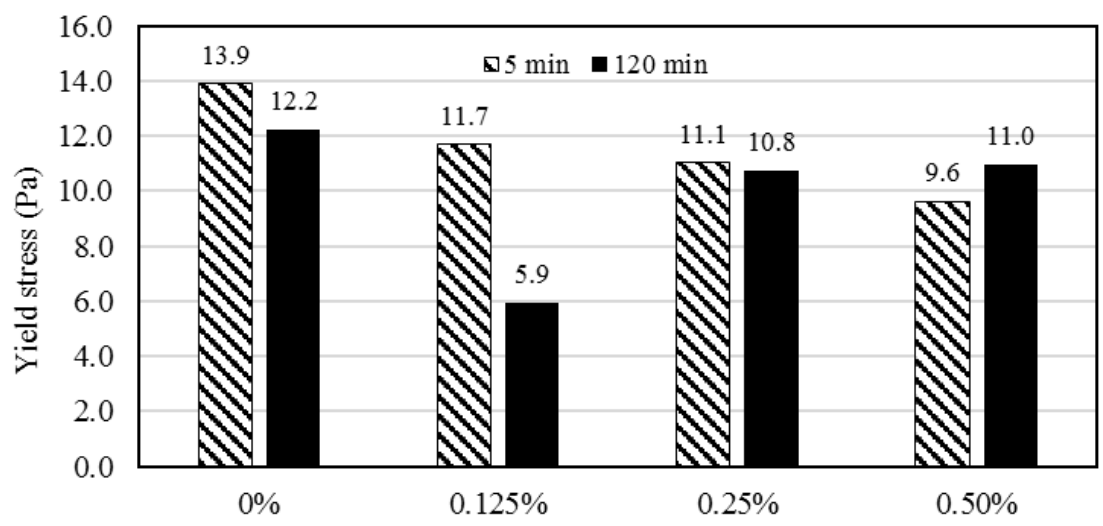

Dosage of MWCNT dispersion (\% by wieght of cement )

Fig.7. Variation of yield stress of nanomodified cement pastes in 5 and 120 min after mixing.

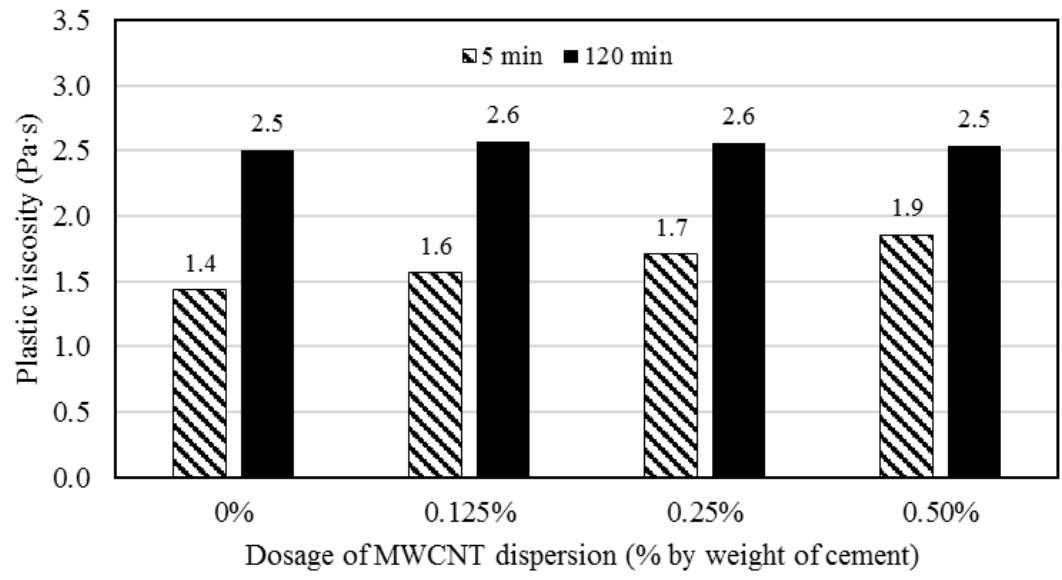

Fig.8. Variation of plastic viscosity of nanomodified cement pastes in 5 and 120 min after mixing.

The yield stress of nanomodified cement paste decreased with increasing the dosage of MWCNT dispersion in the range from 0 to $0.5 \%$ by weight of cement. The effect of MWCNT on yield stress of cement pastes was kept within 120 min after mixing. The maximum decrease of yield stress by $30.9 \%$ in 5 min after mixing was reached for cement paste modified by $0.50 \%$ of MWCNT dispersion by weight of cement in comparison with reference cement paste without MWCNT. The maximum decrease of yield stress by $57.6 \%$ in 120 min after mixing was established for cement paste modified by $0.125 \%$ of MWCNT dispersion. However, this result can demonstrate the mistake in rheological test or not homogeneous distribution of MWCNT dispersion in the volume of cement paste due to the incompatibility of this results with results for other dosage of MWCNT dispersion. Taking this point into account, it is more possible that modification of cement paste by MWCNT dispersion decrease the yield stress approximately by $9.8-11.5 \%$ in comparison with reference cement paste in 120 min after mixing when the dosage of MWCNT dispersion is $0.25 .0 .5 \%$ by weight of cement.

The modification of cement paste by MWCNT dispersion led to the changes of plastic viscosity as well. However, this changes were observed only for samples tested in $5 \mathrm{~min}$ after mixing. The plasticizing effect did not identify within $120 \mathrm{~min}$ after mixing. The increase of the MWCNT dispersion dosage from 0 to $0.50 \%$ increase the values of plastic 
viscosity of cement pastes. The maximum increase by $35.7 \%$ was obtained for cement paste modified by $0.50 \%$ MWCNT dispersion by weight of cement. The plastic viscosity of cement pastes increased about 1.5-2.0 times in $120 \mathrm{~min}$ after mixing. The addition of MWCNT did not influence significantly on the plastic viscosity of cement paste in comparison with reference sample in 120 min after mixing.

The changes in rheological behaviour of cement pastes modified by MWCNT dispersion may be linked with small size of nanostructures and their possible steric interactions with cement particles.

\section{Conclusions}

The following conclusions are drawn in this study:

1. The MWCNT dispersion for modification of cement pastes with average particle diameter up to $294 \mathrm{~nm}$ was obtained in the course of research. The duration of ultrasonication about $6 \mathrm{~min}$, the power equal to $400 \mathrm{~W}$ was established as optimal parameter of nano dispersion homogenization. The obtained MWCNT dispersion showed the stability for 1 month of storage.

2. The changes in $\mathrm{pH}$ level and electrical conductivity of MWCNT dispersion were observed in the range from 8.89 to 8.02 and from 2.00 to $2.75 \mathrm{mS} / \mathrm{cm}$, respectively, during the 1 month of storage. The possible reason of these changes can be explained by physicchemical interactions between MWCNT and water molecules.

3. The introduction of MWCNT dispersion led to the changes in rheological properties of cement pastes. The yield stress was decreased by $30.9 \%$ and $9.8 \%$ for cement paste modified by $0.50 \%$ of MWCNT dispersion by weight of cement within 5 min and $120 \mathrm{~min}$ after mixing, respectively. The plastic viscosity of cement paste modified by $0.50 \%$ of MWCNT dispersion by weight of cement was increased by $35.7 \%$ in 5 min after mixing of cement paste and did not change in $120 \mathrm{~min}$ after mixing. The possible reason of observed changes in rheological properties of nanomodified cement pastes is caused by quite small size of nanostructures and their steric interactions with cement particles.

\section{References}

1. K. Kovler, N. Roussel, Cem.Con. Res. 41, 775-792 (2011)

2. I. Mehdipour, A. Kumar, K. H. Khayat, Mat. Des. 127, 54-66 (2017)

3. D. Feys, K. H. Khayat, R. Khatibk, Cem. Con. Comp. 66, 38-46 (2016)

4. J. Dils, V. Boel, G. D. Schutter, Constr. Build. Mat. 41, 455-463 (2013)

5. I. Gonzalez-Taboada, B. Gonzalez-Fonteboa, J. Eiras-Lopez, G. Rojo-Lopez, J. Clean. Prod. 156, 1-18 (2017)

6. M. Dauksys, G. Skripkiunas, Constr. Build. Mat. 83, 53-61 (2015)

7. C. A. Anagnostopoulos, Constr. Build. Mat. 50, 162-168 (2014)

8. Y. Zhang, X. Luo, X. Kong, F. Wang, L. Gao, P. Tech. 330, 219-227 (2018)

9. A. Sobolkina, V. Mechtcherine, V. Khavrus, D. Maier, M. Mende, M. Ritschel, A. Leonhardt, Cem. Con. Comp. 34, 1104-1113 (2012)

10. M.S. Konsta-Gdoutos, P.A. Danoglidis, M.G. Falara, S.F. Nitodas, Cem. Con. Comp. 82, 137-151 (2017)

11. B. Wang, Y. Han, S. Liu, Const. Build. Mat. 46, 8-12 (2013)

12. S. Xu, J. Liu, Q. Li, Const. Build. Mat. 76, 16-23 (2015)

13. F.T. Isfahani, W. Li, E. Redaelli, Cem. Con. Comp. 74, 154-163 (2016) 
14. T. Jarolim, M. Labaj, R. Hela, K. Michnova, Adv. Mat. Sci. Eng. Article ID 7508904 (2016)

15. B. Zou, S. J. Chen, A.H. Korayem, F. Collins, C.M. Wang, W.H. Duan, Carbon 85 212-220 (2015)

16. D.T.R. Figueiredo, A.A.S. Correia, A.A.S, D. Hunkelerc, M.G.B.V Rasteiro, Col. Surf. A: Physicochem. Eng. Asp. 480, 405-412 (2015)

17. S. Hongyu, C. Binmeng, C., L. Bo, T. Shengwen, L. Zongjin, Constr. Build. Mat. 131, 186-194 (2017) 